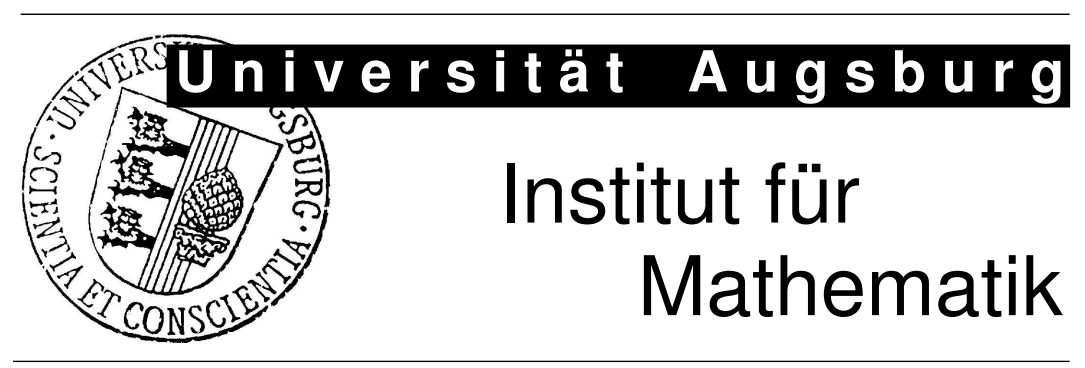

Malte A. Peter, Michael H. Meylan

Water-Wave Scattering by Vast Fields of Bodies

Preprint Nr. 23/2009 - 24. September 2009

Institut für Mathematik, Universitätsstraße, D-86135 Augsburg

http://www.math.uni-augsburg.de/ 


\section{Impressum:}

\section{Herausgeber:}

Institut für Mathematik

Universität Augsburg

86135 Augsburg

http://www.math.uni-augsburg.de/pages/de/forschung/preprints.shtml

\section{ViSdP:}

Malte A. Peter

Institut für Mathematik

Universität Augsburg

86135 Augsburg

Preprint: Sämtliche Rechte verbleiben den Autoren (C) 2009 


\title{
WATER-WAVE SCATTERING BY VAST FIELDS OF BODIES
}

\author{
MALTE A. PETER* ${ }^{*}$ AND MICHAEL H. MEYLAN ${ }^{\dagger}$
}

\begin{abstract}
A very efficient solution method to the determination of the linear water-wave scattering by a large number of bodies is presented. Several bodies are assembled in modules, which are grouped in periodic infinite line arrays. Then, using an iterative method, a finite number of these infinite arrays are stacked together. The method to calculate the scattering by the infinite line array of modules of bodies is algebraicly exact while a far-field (or wide-spacing) approximation is used in the calculation of the scattering of a finite stack of arrays. Bloch transmission through doubly periodic arrangements of bodies is discussed and so are averaging techniques to suppress phenomena introduced by the periodicity assumption on the line arrays for the case of more or less randomly distributed bodies. While the method is general and can be used in a variety of situations, the principle application of the method is to calculate the scattering by vast fields of ice floes which occur in the Marginal Ice Zone. Preliminary numerical simulations for floating elastic plates, modelling ice floes, are presented and substantiate the applicability of the method.
\end{abstract}

Key words. Water waves, diffraction, multiple arrays, Marginal Ice Zone, Bloch waves.

AMS subject classifications. 76B15, 86A05, 74F10, 35B10.

1. Introduction. We present a computationally efficient method to calculate the reflection and transmission of water waves by vast fields of bodies within the framework of linear theory. The method is general in the sense that it it is not restricted to certain types of bodies or body geometries. It is the aim to apply this method in the future to approximate the scattering characteristics of the Marginal Ice Zone (a region of broken ice which forms at the boundary of the frozen and open ocean) without having to assume the problem is two-dimensional. The method is general and is also potentially applicable in a variety of other situations, such as scattering by Very Large Floating Structures supported by many columns or by large off-shore wind farms.

The idea is briefly summarised as follows: We calculate the scattering of a large field of bodies by first grouping several bodies into modules and then determining the scattering characteristics of an infinite periodic line array of such modules. The field of bodies is then assembled by placing many infinite line arrays behind one another in a stack. The solution for this stack is found using a wide-spacing approximation. A sketch of the geometry is given in figure 1.1.

Assuming that the scattering by single bodies can be calculated, an interaction theory $[8,26]$ provides an efficient exact algebraic method for calculating the scattering by finitely many bodies. Grouping such an arrangement of bodies into a module, the scattering by an infinite periodic line array of modules is efficiently calculated using the method described in [30]. It is well-known (see [35, 30, 37], e.g.) that the scattered wavefield away from the array consists of plane waves propagating in a finite number of directions, the so-called scattering angles. Generally, such far-field approximations (also called wide-spacing or plane-wave approximations; see [5] for a detailed discussion in two spatial dimensions) require that the spacing is much larger than the

${ }^{*}$ Department of Mathematics, University of Auckland, Private Bag 92019, Auckland 1142, New Zealand. Current address: Institute of Mathematics, University of Augsburg, 86135 Augsburg, Germany and Augsburg Centre for Innovative Technologies, University of Augsburg, Germany. (malte.peter@math . uni-augsburg.de).

${ }^{\dagger}$ Department of Mathematics, University of Auckland, Private Bag 92019, Auckland 1142, New Zealand (meylan@math.auckland.ac.nz). 


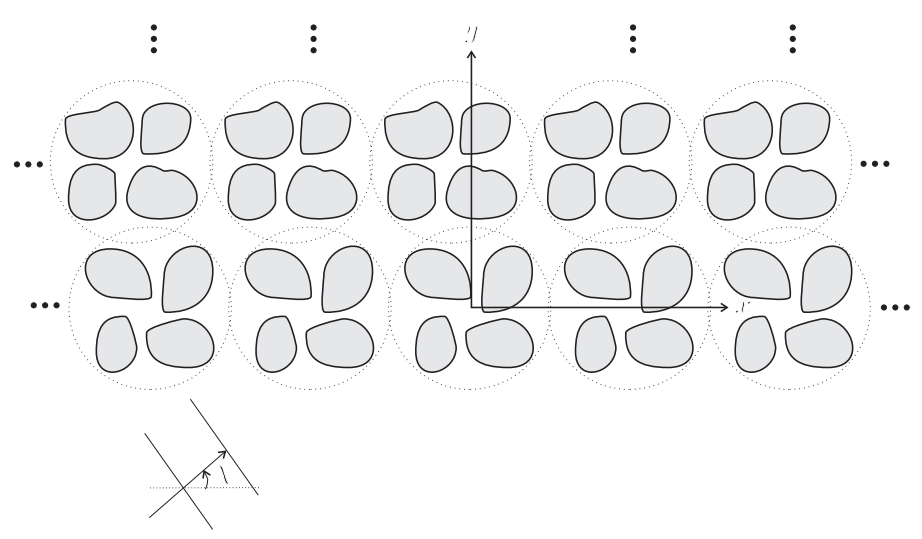

FIG. 1.1. Sketch of the geometry (plan view). Many periodic arrays of modules form the stack.

incident wavelength and the size of the structure (see the monographs $[13,15]$ for discussions) but it was illustrated in [30] that this far-field approximation is accurate even near the array in most situations and we will give some more evidence here. We then present an explicit iterative method allowing the scattering characteristics of a stack of many arrays placed one behind the other to be calculated. The idea of using the far-field approximation to couple multiple arrays was used by [4] for water-wave scattering by stacks of circular cylinders and a linear system of equations for the total scattering was developed, the dimension of which depends linearly on the number of the arrays included. The same idea was used in the context of acoustic scattering in [24] and for electromagnetic scattering in [20, 19], both of which give an iterative method for the coupling of the arrays. All of these works only consider scattering by circular cylinders, which leads to several simplifications not applicable in the general setting considered here, and it seems that they have been developed independently from each other.

The only restriction of the method presented here is that it requires that the arrays have the same periodic spacing and that the spacing between the arrays is not too small so that the far-field approximation is valid. It does not require that the arrays are identical or aligned nor that the spacing between arrays is constant. If the incident wavelength is very large compared to the body spacing, homogenisation techniques might be more appropriate and we refer to [7] as well as the review article [18] for more information in this direction in the water-wave context.

It is well-known that periodic structures may exhibit certain resonance phenomena. For periodic line arrays, scattering angles are aligned with the array axis in some situations or Rayleigh-Bloch waves may travel up or down the array [31, 27] (the latter of which cannot be excited by an incident wave, however). Doubly periodic structures (i.e. structures that extend periodically in both horizontal dimensions) can support Bragg resonance, where phases of reflected waves are the same so that reflected waves interfere constructively, and may admit so-called passing bands and stopping bands, for which waves propagate through the array without change of amplitude or for which the wave amplitude increases or decays (cf. [17, 7] and the monograph [13], e.g.). Often, these phenomena have an implication for the corresponding semi-infinite or finite structure but they require the otherwise periodic arrangement of the bodies. We only briefly discuss the calculation of the Bloch transmission coefficient, as this may be of interest for approximating the stopping and passing bands for regu- 
larly spaced structures. However, since we are particularly interested in more or less random arrangements of bodies in order to apply this method to scattering in the Antarctic Marginal Ice Zone (MIZ) in the future, we do not investigate any other such phenomena in great detail but discuss averaging methods to suppress effects artificially introduced by the periodicity instead.

The MIZ (Marginal Ice Zone) is an interfacial region which forms at the boundary of the open and frozen oceans. It consists of vast fields of ice floes, which scatter and attenuate the incoming ocean waves. It is of great importance to climate research to understand wave propagation and scattering and wave-induced break up in the MIZ (see [34, 33] for more information). Models for wave propagation were developed by [36] based on two-dimensional approximation and this model was developed further by [10] and shown to give reasonable agreement with measurements. Three dimensional models based on the Boltzmann equation were developed by [16, 25, 23, 22] but these models have proved too complicated to extract geophysical data and so far have never been used to make comparisons with experiments. We aim to use the method presented here to create a hybrid two-three dimensional model which will be sufficiently computationally efficient that we can determine the attenuation coefficients for a range of conditions and to compare this to two-dimensional theory [10] and to experimental measurements [32]. We also note that the present method does not depend on the model used for the individual floe. We use here the ice-floe model developed by [21], which is based on an assumption of shallow draft. However, the generality of the method allows to use other ice-floe models, for example accounting for finite draught as modelled by [2].

The paper is organised as follows: The problem is formulated in detail in $\S 2$ including the introduction of the required eigenfunctions expansions and diffraction transfer operators. The solution method for a module of bodies is discussed in $\S 3$ and for an infinite periodic line array of such modules in $\S 4$, which also contains a description of the far field and the definition of the reflection and transmission matrices of such an array. The iterative method of stacking up multiple arrays is given in $\S 5$. In $\S 6$, Bloch transmission in doubly periodic stacks is discussed as well as averaging strategies to suppress artefacts introduced by the periodicity assumption. Numerical experiments are conducted in $\S 7$, including simulation results for bottom-mounted cylinders and ice floes. A summary of the results and discussion is given in $\S 8$.

We also note that a preliminary summary of this paper has appeared in [28].

2. Statement of the problem and mathematical formulation. We consider the water-wave scattering of a plane wave by vertically non-overlapping bodies. The ambient plane wave is assumed to travel in the given direction $\chi \in(-\pi, \pi)$ where $\chi$ is measured with respect to the $x$-axis. Let $\left(r_{j}, \theta_{j}, z\right)$ be the local cylindrical coordinates of the $j$ th body, $\Delta_{j}$. The global coordinates, centred at the origin, are denoted by $(x, y, z)$ (Cartesian) or $(r, \theta, z)$ (cylindrical). A sketch of the geometry is given in figure 1.1.

The equations of motion for the water are derived from the linearised inviscid theory. Under the assumption of irrotational motion, the velocity-vector field of the water can be written as the gradient field of a scalar velocity potential $\Phi$. Assuming that the motion is time-harmonic with radian frequency $\omega$, the velocity potential can be expressed as the real part of a complex quantity,

$$
\Phi(\mathbf{y}, t)=\operatorname{Re}\left\{\phi(\mathbf{y}) \mathrm{e}^{-\mathrm{i} \omega t}\right\} .
$$

To simplify notation, $\mathbf{y}=(x, y, z)$ always denotes a point in the water, which is 
assumed to be of constant finite depth $d$, while $\mathbf{x}$ always denotes a point of the undisturbed water surface assumed at $z=0$.

Writing $\alpha=\omega^{2} / g$ where $g$ is the gravitational acceleration, the potential $\phi$ has to satisfy the standard boundary-value problem

$$
\begin{aligned}
\nabla_{\mathbf{y}}^{2} \phi & =0, & & \mathbf{y} \in D, \\
\partial_{z} \phi & =\alpha \phi, & & \mathbf{x} \in \Gamma^{\mathrm{f}}, \\
\partial_{z} \phi & =0, & & \mathbf{y} \in D, z=-d,
\end{aligned}
$$

where $D=\left(\mathbb{R}^{2} \times(-d, 0)\right) \backslash \bigcup_{j} \bar{\Delta}_{j}$ is the domain occupied by the water and $\Gamma^{\mathrm{f}}$ is the free water surface. At the immersed body surface $\Gamma_{j}$ of $\Delta_{j}$, the water velocity potential has to equal the normal velocity of the body $\mathbf{v}_{j}$,

$$
\partial_{n} \phi=\mathbf{v}_{j}, \quad \mathbf{y} \in \Gamma_{j}
$$

A further relationship between the potential and its normal derivative on the body surface is required if $\mathbf{v}_{j}$ depends of $\phi$, and this comes from the equation of motion for the body. Moreover, a radiation condition is imposed ensuring that there are only outgoing waves from each scatterer and we denote the ambient incident potential by $\phi^{\text {In }}$. The positive wavenumber $k$ is related to $\alpha$ by the dispersion relation

$$
\alpha=k \tanh k d
$$

and the values of $k_{m}, m>0$, are given as positive real roots of the dispersion relation

$$
\alpha+k_{m} \tan k_{m} d=0
$$

For ease of notation, we write $k_{0}=-\mathrm{i} k$. Note that $k_{0}$ is a (purely imaginary) root of (2.4).

2.1. Eigenfunction expansion of the potential. The scattered potential of a body $\Delta_{j}$ can be expanded in singular cylindrical eigenfunctions,

$$
\phi_{j}^{\mathrm{S}}\left(r_{j}, \theta_{j}, z\right)=\sum_{m=0}^{\infty} f_{m}(z) \sum_{\mu=-\infty}^{\infty} A_{m \mu}^{j} K_{\mu}\left(k_{m} r_{j}\right) \mathrm{e}^{\mathrm{i} \mu \theta_{j}}
$$

with discrete coefficients $A_{m \mu}^{j}$, where

$$
f_{m}(z)=\frac{\cos k_{m}(z+d)}{\cos k_{m} d} .
$$

The incident potential upon body $\Delta_{j}$ can be also be expanded in regular cylindrical eigenfunctions,

$$
\phi_{j}^{\mathrm{I}}\left(r_{j}, \theta_{j}, z\right)=\sum_{n=0}^{\infty} f_{n}(z) \sum_{\nu=-\infty}^{\infty} D_{n \nu}^{j} I_{\nu}\left(k_{n} r_{j}\right) \mathrm{e}^{\mathrm{i} \nu \theta_{j}}
$$

with discrete coefficients $D_{n \nu}^{j}$. In these expansions, $I_{\nu}$ and $K_{\nu}$ denote the modified Bessel functions of the first and second kind, respectively, both of order $\nu$ as defined in [1]. Note that in (2.5) (and (2.7)) the term for $m=0(n=0)$ corresponds to the 
propagating modes while the terms for $m \geq 1(n \geq 1)$ correspond to the evanescent modes.

In what follows, it is necessary to represent the ambient wavefield in the eigenfunction expansion (2.7). This can be accomplished as follows. In Cartesian coordinates centred at the origin, the ambient wavefield is given by

$$
\phi^{\operatorname{In}}(x, y, z)=\frac{A g}{\omega} f_{0}(z) \mathrm{e}^{\mathrm{i} k(x \cos \chi+y \sin \chi)},
$$

where $A$ is the amplitude (in displacement) and $\chi \in(-\pi, \pi)$ is the angle between the $x$-axis and the direction in which the wavefield travels (also cf. figure 1.1). This expression can be written in the eigenfunction expansion centred at the origin as

$$
\phi^{\mathrm{In}}(r, \theta, z)=\frac{A g}{\omega} f_{0}(z) \sum_{\nu=-\infty}^{\infty} \mathrm{e}^{\mathrm{i} \nu(\pi-\chi)} I_{\nu}\left(k_{0} r\right) \mathrm{e}^{\mathrm{i} \nu \theta}
$$

so that the ambient incident wavefield has coefficients

$$
\tilde{D}_{n \nu}= \begin{cases}\frac{A g}{\omega}(-1)^{\nu} \mathrm{e}^{-\mathrm{i} \nu \chi}, & n=0 \\ 0, & n>0\end{cases}
$$

in the expansion (2.7). Note that the evanescent coefficients are all zero due to the propagating nature of the ambient wave.

2.2. Diffraction transfer operators. In what follows, we make extensive use of diffraction transfer operators, sometimes referred to as T-matrices. In general, it is possible to relate the total incident and scattered partial waves for any structure through the diffraction characteristics of that body in isolation. For each body $\Delta_{j}$, there exists a diffraction transfer operator $B^{j}$ that relates the coefficients of the incident and scattered partial waves, such that

$$
A_{m \mu}^{j}=\sum_{n=0}^{\infty} \sum_{\nu=-\infty}^{\infty} B_{m n \mu \nu}^{j} D_{n \nu}^{j}
$$

where $A^{j}$ contains the amplitudes of the scattered modes due to the incident modes of amplitude $D^{j}$. The idea of the diffraction transfer operator is not restricted to a single structure. We can thus associate such an operator with a module. More general information on diffraction transfer operators can be found in the monograph [15] (referred to as T-matrices therein).

Assuming methods for solving the standard scattering problem for each body involved are available, the corresponding (truncated) diffraction transfer operator can be calculated numerically. Different methods are outlined in [6, 9, 30]. For some special cases, analytic representations exist. For example, for a rigid bottom-mounted circular cylinder of radius $a$, the elements of the diffraction transfer operator are

$$
B_{m n \mu \nu}= \begin{cases}-I_{\mu}^{\prime}\left(k_{0} a\right) / K_{\mu}^{\prime}\left(k_{0} a\right), & 0 \leq m=n<\infty,-\infty<\mu=\nu<\infty \\ 0, & \text { otherwise }\end{cases}
$$

It is also worth noting that the change in the diffraction transfer operator induced by a rotation of the body about its mean-centre position is particularly simple [26]. 
3. Scattering by a finite number of bodies and grouping into modules. The scattering properties of a finite number of bodies can be calculated in many different ways, for example by using the finite element method, which involves discretising all body surfaces, or, more efficiently, using an interaction theory $[8,26]$. For our purposes, the scattered wavefield needs to be represented in terms of eigenfunction expansions (2.5) in order to allow the scattering properties to be described by a single diffraction transfer operator. We briefly summarise how this can be achieved using the interaction theory. Note that the idea of using the interaction theory to group several bodies into modules has been successfully applied previously $[9,3]$.

The interaction theory works by developing a system of equations for the unknown coefficients (in the expansion (2.5)) of the scattered wavefields of all bodies. This system of equations is based on transforming the scattered potential of $\Delta_{j}$ into an incident potential upon $\Delta_{l}, j, l=1, \ldots, N, j \neq l$. Doing this for all bodies simultaneously, and relating the incident and scattered potential for each body, a system of equations for the unknown coefficients is developed.

The scattered potential $\phi_{j}^{\mathrm{S}}$ of body $\Delta_{j}$ needs to be represented in terms of the incident potential $\phi_{l}^{\mathrm{I}}$ upon $\Delta_{l}, j \neq l$ and this can be achieved using Graf's addition theorem (eq. 9.1.79 in [1]). Since the expansion of the scattered and incident potential in cylindrical eigenfunctions is only valid outside the escribed cylinder of each body, the escribed cylinder of each body may not contain any other body.

Making use of the eigenfunction expansion as well as Graf's addition theorem, the scattered potential of $\Delta_{j}$ (cf. (2.5)) can be expressed in terms of the incident potential in the local coordinates of $\Delta_{l}$ as

$$
\begin{aligned}
& \phi_{j}^{\mathrm{S}}\left(r_{l}, \theta_{l}, z\right) \\
& =\sum_{m=0}^{\infty} f_{m}(z) \sum_{\nu=-\infty}^{\infty}\left[\sum_{\tau=-\infty}^{\infty} A_{m \tau}^{j}(-1)^{\nu} K_{\tau-\nu}\left(k_{m} R_{j l}\right) \mathrm{e}^{\mathrm{i}(\tau-\nu) \varphi_{j l}}\right] I_{\nu}\left(k_{m} r_{l}\right) \mathrm{e}^{\mathrm{i} \nu \theta_{l}},
\end{aligned}
$$

where $\left(R_{j l}, \vartheta_{j l}\right)$ are the coordinates of the mean-centre position of the $l$ th body in terms of the coordinate system of the $j$ th body. Let $\hat{D}_{n \nu}^{l}$ denote the coefficients of an ambient incident wavefield in the incoming eigenfunction expansion for $\Delta_{l}$ (cf. (2.7)). The total incident wavefield upon body $\Delta_{l}$ can now be expressed as in terms of the coefficients

$$
D_{n \nu}^{l}=\hat{D}_{n \nu}^{l}+\sum_{\substack{j=1 \\ j \neq l}}^{N} \sum_{\tau=-\infty}^{\infty} A_{n \tau}^{j}(-1)^{\nu} K_{\tau-\nu}\left(k_{n} R_{j l}\right) \mathrm{e}^{\mathrm{i}(\tau-\nu) \vartheta_{j l}}
$$

in the expansion (2.7).

Using the diffraction transfer operator, the substitution of (3.2) into (2.10) gives the required equations to determine the coefficients of the scattered wavefields of all bodies,

$$
A_{m \mu}^{l}=\sum_{n=0}^{\infty} \sum_{\nu=-\infty}^{\infty} B_{m n \mu \nu}^{l}\left[\hat{D}_{n \nu}^{l}+\sum_{\substack{j=1 \\ j \neq l}}^{N} \sum_{\tau=-\infty}^{\infty} A_{n \tau}^{j}(-1)^{\nu} K_{\tau-\nu}\left(k_{n} R_{j l}\right) \mathrm{e}^{\mathrm{i}(\tau-\nu) \vartheta_{j l}}\right],
$$

$m \in \mathbb{N}, \mu \in \mathbb{Z}, l=1, \ldots, N$.

For a given incident wave having coefficients $\hat{D}_{n \nu}^{l}$ in the local expansion (2.7), the solution of (3.3) gives the coefficients $A_{m \mu}^{l}$ of the scattered wavefield around each body in the expansion (2.5). 
In order to obtain the diffraction transfer operator of the module made up of bodies $\Delta_{j}, j=1, \ldots, N$, system (3.3) needs to be solved for all possible incident waves of unit amplitude with respect to the origin (i.e. for $D_{n \nu}=1$ for one $(n, \nu)$ at a time and zero for the others). The conversion to the local coordinates of $\Delta_{l}$ is given by

$$
\hat{D}_{n \nu}^{l}=\sum_{\tau=-\infty}^{\infty} D_{n \tau} I_{\tau-\nu}\left(k_{n} R_{l}\right) \mathrm{e}^{\mathrm{i}(\tau-\nu) \vartheta_{l}}
$$

where $\left(R_{l}, \vartheta_{l}\right)$ is the mean-centre position of $\Delta_{l}$ in polar coordinates and where we have again utilised Graf's addition theorem. Solving (3.3) for each $D_{n \nu}$, the resulting total scattered wave of the modules (with respect to the origin) is similarly given by

$$
A_{m \mu}^{\mathrm{tot}}=\sum_{l=1}^{N} \sum_{\tau=-\infty}^{\infty} A_{m \tau}^{l} I_{\mu-\tau}\left(k_{m} R_{l}\right) \mathrm{e}^{-\mathrm{i}(\mu-\tau) \vartheta_{l}}
$$

from which the elements of the diffraction transfer operator of the module can be read off directly.

4. Scattering by a periodic line array of bodies. In the same way as in the previous section, the interaction theory can be used to derive a system of equations for the periodic line array of identical modules, where the modules have mean-centre positions $(j R, 0), j \in \mathbb{Z}$, and the same diffraction transfer operators $M$ (see [30] for details). In this case, system of equations (3.3) becomes

$$
A_{m \mu}^{l}=\sum_{n=0}^{\infty} \sum_{\nu=-\infty}^{\infty} M_{m n \mu \nu}\left[\tilde{D}_{n \nu}^{l}+\sum_{\substack{j=-\infty \\ j \neq l}}^{\infty} \sum_{\tau=-\infty}^{\infty} A_{n \tau}^{j}(-1)^{\nu} K_{\tau-\nu}\left(k_{n}|j-l| R\right) \mathrm{e}^{\mathrm{i}(\tau-\nu) \varphi_{j-l}}\right],
$$

$m \in \mathbb{N}, l, \mu \in \mathbb{Z}$, where the angles $\varphi_{n}$ account for the difference in direction depending if the $j$ th module is located to the left or to the right of the $l$ th module and are defined by

$$
\varphi_{n}= \begin{cases}\pi, & n>0 \\ 0, & n<0\end{cases}
$$

Because of the periodicity of the geometry and of the incident wave, the coefficients $A_{m \mu}^{l}$ can be written as $A_{m \mu}^{l}=P_{l} A_{m \mu}^{0}=P_{l} A_{m \mu}$, say, where $P_{l}=\mathrm{e}^{\mathrm{i} l R k \cos \chi}$. The same can be done for the coefficients of the incident ambient wave, i.e. $\tilde{D}_{n \nu}^{l}=P_{l} \tilde{D}_{n \nu}$. Noting that $P_{l}^{-1}=P_{-l}$ and $P_{j} P_{l}=P_{j+l},(4.1)$ simplifies to

$$
A_{m \mu}=\sum_{n=0}^{\infty} \sum_{\nu=-\infty}^{\infty} M_{m n \mu \nu}\left[\tilde{D}_{n \nu}+(-1)^{\nu} \sum_{\tau=-\infty}^{\infty} A_{n \tau} \sum_{\substack{j=-\infty \\ j \neq l}}^{\infty} P_{j-l} K_{\tau-\nu}\left(k_{n}|j-l| R\right) \mathrm{e}^{\mathrm{i}(\tau-\nu) \varphi_{j-l}}\right] .
$$

Introducing the constants

$$
\sigma_{\nu}^{n}=\sum_{\substack{j=-\infty \\ j \neq l}}^{\infty} P_{j-l} K_{\nu}\left(k_{n}|j-l| R\right) \mathrm{e}^{\mathrm{i} \nu \varphi_{j-l}}=\sum_{j=1}^{\infty}\left(P_{-j}+(-1)^{\nu} P_{j}\right) K_{\nu}\left(k_{n} j R\right)
$$


which can be evaluated separately since they do not contain any unknowns, the problem reduces to

$$
A_{m \mu}=\sum_{n=0}^{\infty} \sum_{\nu=-\infty}^{\infty} M_{m n \mu \nu}\left[\tilde{D}_{n \nu}+(-1)^{\nu} \sum_{\tau=-\infty}^{\infty} A_{n \tau} \sigma_{\tau-\nu}^{n}\right] .
$$

The efficient computation of the constants $\sigma_{\nu}^{0}$ is not trivial as the sum in (4.2) is not absolutely convergent owing to the slow decay of the modified Bessel function of the second kind for large imaginary argument (the terms in the sum decay like $j^{-1 / 2} \mathrm{e}^{\mathrm{i} j \theta}$ for some $\theta$ ). Appropriate methods for the computation of the $\sigma_{\nu}^{0}$ are outlined in [30] based on results of [11]. The calculation of the constants $\sigma_{\nu}^{n}, n \neq 0$, is easy, however, since the modified Bessel function of the second kind decays exponentially for large real argument.

4.1. The far field. In this section, the far field is examined which describes the scattering far away from the array. The derivation can be found in [30] and is equivalent to that of [35] for electromagnetic scattering. First, we define the scattering angles, which give the directions of propagation of plane scattered waves far away from the array. Letting $p=2 \pi / R$, define the scattering angles $\chi_{m}$ by

$$
\chi_{m}=\arccos \left(\psi_{m} / k\right) \quad \text { where } \quad \psi_{m}=k \cos \chi+m p
$$

and write $\psi$ for $\psi_{0}$. Also note that $\chi_{0}=\chi$ by definition. If $\left|\psi_{m}\right|<k$, i.e. if

$$
-1<\cos \chi+\frac{m p}{k}<1
$$

we say that $m \in \mathcal{M}$ and then $0<\chi_{m}<\pi$. It turns out (see below) that these angles $\left( \pm \chi_{m}\right.$ for $\left.m \in \mathcal{M}\right)$ are the directions in which plane waves propagate away from the array.

The only terms which contribute to the far field are those for which $\left|\psi_{m}\right|<k$. Thus, as $y \rightarrow \pm \infty$, the far field consists of a set of plane waves propagating in the directions $\theta= \pm \chi_{m}$ :

$$
\phi \sim \phi^{\mathrm{In}}+\frac{\pi \mathrm{i}}{k R} f_{0}(z) \sum_{m \in \mathcal{M}} \frac{1}{\sin \chi_{m}} \mathrm{e}^{\mathrm{i} k r \cos \left(\theta \mp \chi_{m}\right)} \sum_{\mu=-\infty}^{\infty} A_{0 \mu} \mathrm{e}^{ \pm \mathrm{i} \mu \chi_{m}} .
$$

From (4.5) the amplitudes of the scattered waves for each scattering angle $\pm \chi_{m}$ are given in terms of the coefficients $A_{0 \mu}$ by

$$
A_{m}^{ \pm}=\frac{\pi \mathrm{i}}{k R} \frac{1}{\sin \chi_{m}} \sum_{\mu=-\infty}^{\infty} A_{0 \mu} \mathrm{e}^{ \pm \mathrm{i} \mu \chi_{m}}
$$

It is implicit in all of the above that $\sin \chi_{m} \neq 0$ for any $m$. If $\sin \chi_{m}=0$ then we have the special situation, where one of the scattered plane waves propagates along the array. We will not consider this resonant case here, in which the scattered field is dominated by waves travelling along the array, either towards $x=\infty$ (if $\chi_{m}=0$ ) or towards $x=-\infty$ (if $\chi_{m}=\pi$ ), and refer to [14] for details. In particular, this resonance is an artefact of the periodicity assumption. 
4.2. Reflection and transmission matrices. For given $k, R$ and $\chi$, the farfield scattering characteristics of a line array $L_{j}$ are described by the coefficients $A_{m}^{-}$ and $A_{m}^{+}$, calculated for unit incident potential. If $\chi$ is positive, then the amplitudes of the reflected and transmitted waves travelling away from the array are given by $A_{m}^{-}$and $\delta_{m 0}+A_{m}^{+}$, respectively. Analogously, for $\chi$ negative, the amplitudes of the reflected and transmitted waves respectively are $A_{m}^{+}$and $\delta_{m 0}+A_{m}^{-}$. (Note that the $A_{m}^{ \pm}$may be different for $+\chi$ and $-\chi$.)

Thus, for given $k, R$ and $\chi$, we define the reflection and transmission matrices

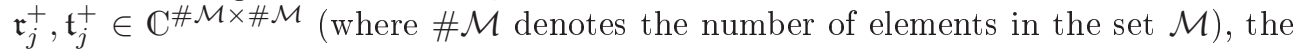
columns of which contain the coefficients $A_{m}^{-}$and $\delta_{m 0}+A_{m}^{+}$, respectively, calculated for each incident angle $+\left|\chi_{n}\right|$. These matrices completely describe the far-field characteristics of the array for the incident wave of angle $+|\chi|$. Analogously, we define $\mathfrak{r}_{j}^{-}$and $\mathfrak{t}_{j}^{-}$having elements $A_{m}^{-}$and $\delta_{m 0}+A_{m}^{+}$, respectively, calculated for each incident angle $-\left|\chi_{n}\right|$, for the same situation but with incident angle $-|\chi|$. The classical scattering matrix is then given by

$$
\left[\begin{array}{ll}
\mathfrak{r}_{j}^{-} & \mathfrak{t}_{j}^{+} \\
\mathfrak{t}_{j}^{-} & \mathfrak{r}_{j}^{+}
\end{array}\right]
$$

Note that the only difference in calculating the elements of $\mathfrak{r}_{j}^{+}$and $\mathfrak{t}_{j}^{+}$compared to $\mathfrak{r}_{j}^{-}$and $\mathfrak{t}_{j}^{-}$is the different right-hand side in (4.3) as one has to use ambient-wave coefficients $\tilde{D}$ in one case and the complex conjugated ones $\tilde{D}^{*}$ in the other. Thus, when calculating the reflection and transmission matrices for given $\chi$, there is very little extra computational cost to compute them for $-\chi$ additionally.

If the array is up-down symmetric (i.e. w.r.t. the $y$-axis), it does not matter whether the incident wave travels in direction $+|\chi|$ or $-|\chi|$ and, in this case, the +and --reflection and transmission matrices are identical, i.e. $\mathfrak{r}_{j}^{-}=\mathfrak{r}_{j}^{+}$and $\mathfrak{t}_{j}^{-}=\mathfrak{t}_{j}^{+}$.

It is useful to know how the reflection and transmission matrices change if the array undergoes a translation such that the mean-centre position of the zeroth body (originally located at $(0,0))$ is shifted to lie at some new position $(x, y)$. Writing $P=\left\lceil\exp \left(\mathrm{i} k x \cos \chi_{m}\right)\right\rfloor$ and $Q=\left\lceil\exp \left(\mathrm{i} k y \sin \chi_{m}\right)\right\rfloor$, where $\left\lceil a_{m}\right\rfloor$ is a diagonal matrix with diagonal elements $a_{m}$, the translation of the array causes the ambient wave incident on the array travelling in positive direction to be phase shifted by $P Q$. In terms of coordinates centred at $(x, y)$, the reflection and transmission matrices of the shifted array are thus given by $\mathfrak{r}_{j}^{+} Q P$ and $\mathfrak{t}_{j}^{+} Q P$. Keeping in mind that the reflected and transmitted waves travel in opposite direction with respect to the $y$-coordinate but in the same direction with respect to the $x$-coordinate, changing the coordinates back to the origin gives

$$
P^{-1} Q \mathfrak{r}_{j}^{+} Q P \quad \text { and } \quad P^{-1} Q^{-1} \mathfrak{t}_{j}^{+} Q P
$$

as the reflection and transmission matrices of the translated array. As expected, a shift in the $x$-direction by a multiple of the array spacing $R$ leaves the matrices unchanged. Analogous formulas hold for $\mathfrak{r}_{j}^{-}$and $\mathfrak{t}_{j}^{-}$but are not required in what follows.

5. Scattering by multiple line arrays. It was found in [30] that in most situations the far-field approximation is very good even near the line array and some numerical experiments confirming this observation are given in $\S 7$. Based on ideas of Mulholland and Heckl [24] for acoustic scattering and McPhedran et al. [20, 19] for electromagnetic scattering, we present an efficient iterative method to stack up a finite number of periodic line arrays assuming that the far-field approximation is 
accurate for the considered stack spacing. It is noteworthy that the previous works only consider arrays of circular cylinders and thus arrays having up-down symmetry.

It is easy to see from (4.4) that, once $k, R$ and $\chi$ are fixed, an incident plane wave making an angle $\chi_{l}$ for a $l \in \mathcal{M}$ will results in transmitted and reflected waves travelling in the directions $\pm \chi_{m}, m \in \mathcal{M}$. Thus, for a stack of arrays of the same horizontal spacing $R$, only waves in the directions $\pm \chi_{m}, m \in \mathcal{M}$, need to be taken into account.

For given $k, R$ and $\chi$, the scattering characteristics of a line array $L_{j}$ are completely described by the reflection and transmission matrices $\mathfrak{r}_{j}^{ \pm}, \mathfrak{t}_{j}^{ \pm} \in \mathbb{C} \# \mathcal{M} \times \# \mathcal{M}$ defined in §4.2. Assuming that the reflection and transmission matrices for a stack of $n-1$ arrays is already known, we derive here how to add on an $n$th array. To keep things as simple as possible, we only consider ambient waves of given incident angle $\chi \in(0, \pi)$ on the stack. Note that this implies no restriction of generality.

Let $\mathfrak{R}_{n-1}=\mathfrak{R}_{n-1}^{+}$and $\mathfrak{T}_{n-1}=\mathfrak{T}_{n-1}^{+}$respectively be the reflection and transmission matrices of the stack consisting of $n-1$ arrays and let $s_{n}>0$ be the (vertical) spacing between the stack and the array to be added on. The phase shift due to moving the stack along the $y$-axis such that the array in the stack, whose axis used to coincide with the $x$-axis, is now on the line $y=s_{n}$ is encoded in the diagonal matrix $Q_{n}=\left\lceil\exp \left(\mathrm{i} k s_{n} \sin \chi_{m}\right)\right\rfloor(\mathrm{cf} . \S 4.2)$. The reflection and transmission matrices of the shifted stack are given by $Q_{n} \Re_{n-1} Q_{n}$ and $Q_{n}^{-1} \mathfrak{T}_{n-1} Q_{n}$, respectively. Now, place a line array characterised by matrices $\mathfrak{r}_{n}^{ \pm}$and $\mathfrak{t}_{n}^{ \pm}$on the $x$-axis as illustrated in figure 5.1. Assuming that the far-field approximation holds, the amplitudes of the waves travelling in between the old stack and the new array are

$$
\begin{aligned}
& f^{-}=Q_{n} \Re_{n-1} Q_{n} f^{+}, \\
& f^{+}=\mathfrak{t}_{n}^{+} \delta+\mathfrak{r}_{n}^{-} f^{-}
\end{aligned}
$$

with an incident wave field $\delta$ on the array. Furthermore, we have

$$
\begin{aligned}
\mathfrak{R}_{n} \delta & =\mathfrak{r}_{n}^{+} \delta+\mathfrak{t}_{n}^{-} f^{-}, \\
\mathfrak{T}_{n} \delta & =Q_{n}^{-1} \mathfrak{T}_{n-1} Q_{n} f^{+} .
\end{aligned}
$$

Eliminating $f^{-}$and $f^{+}$, we find that the total reflection and transmission matrices of the stack composed of $n$ arrays is given by

$$
\begin{aligned}
\mathfrak{R}_{n} & =\mathfrak{r}_{n}^{+}+\mathfrak{t}_{n}^{-} Q_{n} \mathfrak{R}_{n-1} Q_{n}\left(\mathrm{I}-\mathfrak{r}_{n}^{-} Q_{n} \Re_{n-1} Q_{n}\right)^{-1} \mathfrak{t}_{n}^{+}, \\
\mathfrak{T}_{n} & =Q_{n}^{-1} \mathfrak{T}_{n-1} Q_{n}\left(\mathrm{I}-\mathfrak{r}_{n}^{-} Q_{n} \Re_{n-1} Q_{n}\right)^{-1} \mathfrak{t}_{n}^{+} .
\end{aligned}
$$

It is noteworthy that placing the new array in front of the stack is necessary in order to ensure that the scattering characteristics of the stack are only required in one direction. The backward reflection and transmission matrices, i.e. $\mathfrak{R}^{-}$and $\mathfrak{T}^{-}$, can be found by an analogous procedure if required.

A measure for the total transmitted energy through the stack is given by

$$
E^{\text {tot }}=\sum_{m \in \mathcal{M}}\left|\left(\mathfrak{T}_{n}\right)_{m 0}\right|^{2} \sin \chi_{m}
$$

where $\left(\mathfrak{T}_{n}\right)_{m 0}$ is the element $(m, 0)$ of the matrix $\mathfrak{T}_{n-1}$. 


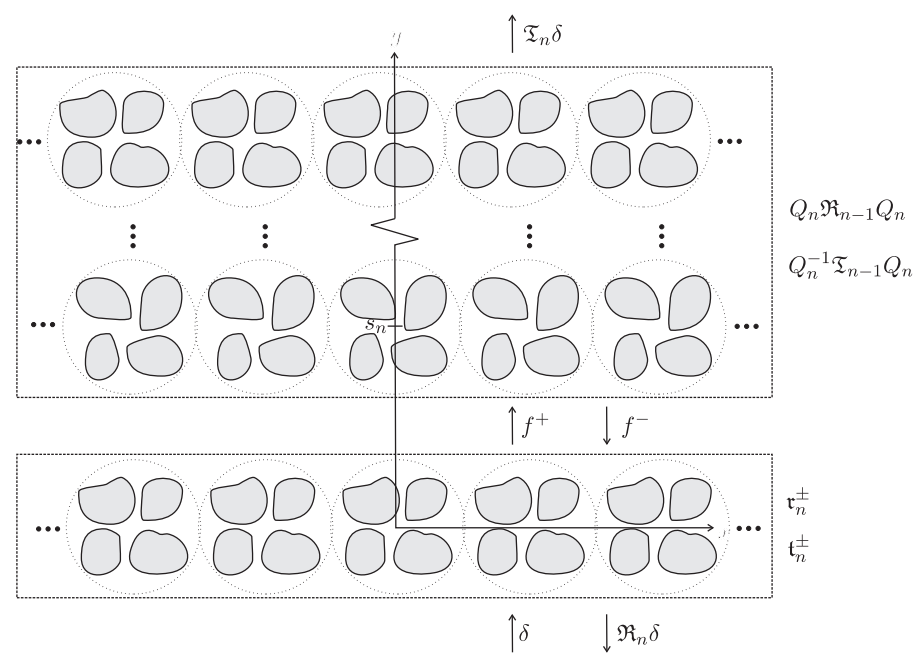

Fig. 5.1. Adding on of the nth single line array to the stack already made up of $n-1$ arrays (plan view). Shifting the old stack along the $y$-axis, the reflection and transmission matrices of the old stack, $\mathfrak{R}_{n-1}$ and $\mathfrak{T}_{n-1}$, become $Q_{n} \Re_{n-1} Q_{n}$ and $Q_{n}^{-1} \mathfrak{T}_{n-1} Q_{n}$ with respect to the origin.

6. Periodic and random arrangements. Some real-world problems involve a periodic arrangement of bodies (some off-shore wind farms or artificial breakwaters, e.g.) while in others the distribution is more or less random (ice floes in the MIZ, e.g.). In the former case, the qualitative scattering behaviour can be well approximated by looking at the corresponding problem for a doubly-periodic array (i.e. an array which extends periodically in both horizontal dimensions). This allows to look for Bloch wave solutions and find the Bloch transmission coefficient, which gives information about passing and stopping bands of the structure. In the latter case, artefacts artificially introduced by periodicity need to be suppressed as much as possible and this is achieved well by averaging over different arrangements. We will consider these two scenarios in this section.

6.1. Bloch waves and Bloch transmission. We first consider the case of a doubly-periodic array, i.e. an array which extends periodically in both horizontal dimensions. In terms of the notation from the previous sections, we assume that, for given $k, R$ and $\chi$, a single line array with reflection and transmission matrices $\mathfrak{r}^{ \pm}, \mathfrak{t}^{ \pm} \in \mathbb{C}^{\# \mathcal{M} \times \# \mathcal{M}}$ as defined in $\S 4.2$ is repeated periodically along the $y$-axis with fixed period (i.e. vertical spacing) $s$. The periodicity of the geometry motivates to look for periodic solutions satisfying

$$
\phi(x, y+s, z)=\mathrm{e}^{\mathrm{i} b s} \phi(x, y, z) .
$$

Such solutions are sometimes called Bloch waves and the factor $\mathrm{e}^{\mathrm{i} b s}$ is the Bloch transmission coefficient. The real part of $b$ encodes the phase change as the wave propagates one period while the imaginary part gives the change in amplitude. Intervals of ambient wavenumbers $k$, for which $b$ is real, are known as passing bands, while those, for which $b$ has non-zero imaginary part, are known as stopping bands.

It is sufficient to consider only a single line array placed on the $x$-axis and to enforce periodicity conditions at $y= \pm s / 2$,

$$
\phi(x, s / 2, z)=\mathrm{e}^{\mathrm{i} b s} \phi(x,-s / 2, z) \quad \text { and } \quad \partial_{y} \phi(x, s / 2, z)=\mathrm{e}^{\mathrm{i} b s} \partial_{y} \phi(x,-s / 2, z) .
$$


A method of determining the value of $b$ for given $k, R$ and $\chi$ can be developed as follows (based on [24]).

We adopt the notation from the previous section for wavefields above and below the array (also cf. fig. 5.1) by respectively writing $\delta^{+}$and $\delta^{-}$for the forward and backward travelling wavefields below the array ( $\delta$ and $\Re_{n-1} \delta$ in the previous section) and $f^{ \pm}$for the corresponding ones above the array. Also keeping in mind that the far-field at $y= \pm s / 2$ has the form (4.5), conditions (6.2) can be expressed as

$$
\mathrm{e}^{\mathrm{i} b s}\left(\delta^{+}+\delta^{-}\right)=Q f^{+}+Q^{-1} f^{-} \quad \text { and } \quad \mathrm{e}^{\mathrm{i} b s}\left(\delta^{+}-\delta^{-}\right)=Q f^{+}-Q^{-1} f^{-} .
$$

Adding and subtracting these two equations gives

$$
\mathrm{e}^{\mathrm{i} b s} \delta^{+}=Q f^{+} \quad \text { and } \quad \mathrm{e}^{\mathrm{i} b s} \delta^{-}=Q^{-1} f^{-} .
$$

From (5.1b) and (5.2a), we also have

$$
f^{+}=\mathfrak{t}^{+} \delta^{+}+\mathfrak{r}^{-} f^{-} \text {and } \delta^{-}=\mathfrak{r}^{+} \delta+\mathfrak{t}^{-} f^{-} .
$$

Combining (6.4) and (6.5) to eliminate $\delta^{-}$and $f^{+}$eventually leads to

$$
\left[\begin{array}{cc}
Q \mathfrak{t}^{+} & Q \mathfrak{r}^{-} \\
-\left(\mathfrak{t}^{-}\right)^{-1} \mathfrak{r}^{+} Q \mathfrak{t}^{+} & -\left(\mathfrak{t}^{-}\right)^{-1} \mathfrak{r}^{+} Q \mathfrak{r}^{-}+\left(Q \mathfrak{t}^{-}\right)^{-1}
\end{array}\right]\left[\begin{array}{c}
\delta^{+} \\
f^{-}
\end{array}\right]=\mathrm{e}^{\mathrm{i} b s}\left[\begin{array}{c}
\delta^{+} \\
f^{-}
\end{array}\right],
$$

i.e. $\mathrm{e}^{ \pm \mathrm{i} b s}$ is found by seeking for eigenvalues of the matrix in equation (6.6) (owing to the symmetry of the problem the eigenvalues appear in pairs of complex conjugates).

6.2. Averaging. Since the method for calculating the scattering by a large stack is computationally cheap, it is particularly suitable if the bodies are arranged more or less randomly and the exact body positions are neither known nor essential. In this case, it is important to suppress effects artificially introduced by the periodicity of the geometry and this can be done by averaging over random samples. This is the strategy, which is aimed for simulating wave scattering in the MIZ.

It is straightforward and computationally very cheap to sample over random choices of distances between the line arrays in a stack (i.e. over vertical spacing $s_{n}$ ). This only requires multiple runs of the iteration (5.3) but ensures non-periodicity in the $y$-direction. The reflection and transmission matrices of a single line array only need to be calculated once.

The next simplest choice is to sample as well over many stacks, each having a different array spacing of the line array (i.e. over horizontal spacing $R$ ). This additionally breaks up the periodicity in the $x$-direction but requires the calculation of new reflection and transmission matrices for each sample. The computational cost is considerably higher than that for the first scheme but it is still of manageable extent as the typically most costly operation, the calculation of the diffraction transfer operators, does not need to be carried out multiple times. As all arrays in one stack have to have the same spacing in order to ensure the same scattering angles, using arrays with different spacings in the same stack is not sensible.

Obviously, there are numerous other possible choices for randomisation and averaging. For example, computationally cheap choices are randomly shifting each line array in the stack by a small distance or randomly rotating modules within each array. We do not follow these ideas here. It will be shown in $\S 7.2$ that the approaches above already give reasonable agreement with experimental data in the case of ice floes in the MIZ. 
It is also noteworthy that, depending on the application, there might be other parameters, over which averaging could be performed. For example, it might be sensible to average over body properties (size, mechanical properties etc.) as well. We restrict here to averaging over random changes in the body positions, that is changes in $s_{n}$ and $R$.

The quantity of main interest when considering scattering by ice floes in the MIZ is the total transmitted energy, which can be measured by the quantity $E^{\text {tot }}$ (cf. (5.4)), and its attenuation. Therefore, we find $E^{\text {tot }}$ as a function of $M$, where $M$ is the number of line arrays in the stack, for given (constant) horizontal array spacing $R$ and vertical spacing $s$ or as an average over $Z$ samples of stacks, each sample yielding an energy $E_{i}^{\text {tot }}, i=1, \ldots, Z$. In this case, $E^{\text {tot }}=\sum_{i=1}^{Z} E_{i}^{\text {tot }} / Z$. If only random choices of distances between the line arrays in a stack are considered, each $E_{i}^{\text {tot }}$ is calculated by choosing each $s_{n}, n=2, \ldots, M$, randomly about a mean $s$ with a given standard deviation. If random choices of array spacings are also required, each $E_{i}^{\text {tot }}$ is calculated by first choosing a common spacing for the line arrays in the $i$ th stack (again as a random choice about a mean $R$ with a given standard deviation) and then choosing each $s_{n}, n=1, \ldots, M$, randomly about a mean $s$ with a given standard deviation as before.

7. Numerical simulations. We present numerical simulations showing some typical results, which can be obtained by the presented method. Two different body types are considered: the rigid bottom-mounted circular cylinder as a simple model for supporting columns of one or more structures or as a breakwater and the floating elastic plate of shallow draft as a model for ice floes. Both models are well-studied and line arrays of such bodies have already been investigated in detail by the authors including validation and comparison with other results [30,27].

The ice floes are modelled as thin elastic plates of shallow draft sitting at the water surface as modelled in [21]. We present results for square ice floes and circular ice floes. The employed solution method for circular ice floes is given in [29].

7.1. Bottom-mounted circular cylinders. We present some simulations for circular rigid bottom-mounted cylinders of radius $a$. This problem is equivalent to two-dimensional acoustic scattering in a medium with constant sound speed $c$. The $z$-dependence can be omitted and the theory above applies with the following modifications:

1. The dispersion relation (2.3) is replaced by $k=\omega / c$ where $c$ is the speed of sound in the medium under consideration and the dispersion relation is (2.4) omitted.

2. All factors $\cos k_{m}(z+d), \cos k_{m} d$ and $f_{0}$ are replaced by 1 .

Note that the first point implies that there are no evanescent modes in this problem, i.e. the sums over $m$ and $n$ in the eigenfunction expansions (2.5) and (2.7), respectively, only contain the terms for $m=0$ and $n=0$. The diffraction transfer operator is given by (2.11). Moreover, we have $k_{0}=-\mathrm{i} \omega / c$. This problem was considered in detail by [12], e.g.

7.1.1. Validity of the far-field approximation. We begin by presenting some computations illustrating how quickly the far-field approximation is accurate in practice. Figure 7.1 shows the real part of the potential along the $y$-axis calculated using 

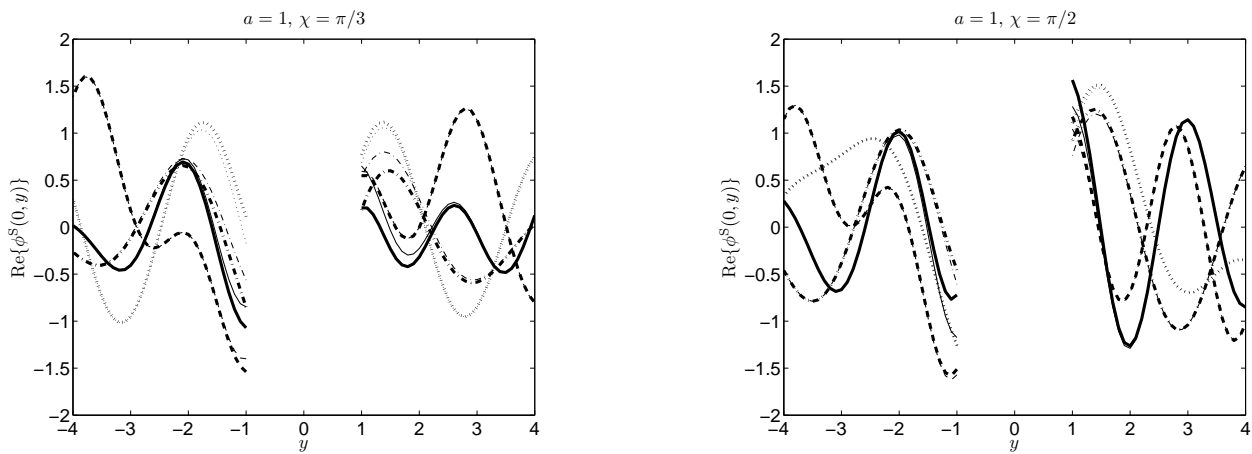

FIG. 7.1. Real part of the potential on the $y$-axis. Thick lines show the full potential calculated via (7.1) while thin lines correspond to the far-field approximation (4.5) for incident angle $\pi / 3$ (left) and $\pi / 2$ (right). The cylinder radius is $a$ is 1 and the other data is given by $\lambda=1.8, R=3$ (solid lines), $\lambda=1.8, R=4$ (dashed lines), $\lambda=2.5, R=3$ (dotted lines) and $\lambda=2.5, R=4$ (dash-dotted lines).

the full solution (thick lines), i.e.

$$
\phi^{\mathrm{S}}=\sum_{j, \mu=-\infty}^{\infty} P_{j} A_{0 \mu} K_{\mu}\left(k_{0} r_{j}\right) \mathrm{e}^{\mathrm{i} \mu \theta_{j}}
$$

and the far-field approximation given by (4.5) (thin lines) for different parameter values. It can be seen that the far-field approximation and the full solution become similar quickly away from the array axis (they are nearly indistinguishable at $y= \pm 2$ for all cases) implying that the approximation is valid even near the array. It can be seen that the approximation is generally more accurate for perpendicular incidence $(\chi=\pi / 2)$. The comparison of the imaginary parts of the potential (not shown) gives analogous results.

7.1.2. Impact of distribution of cylinder radii. We investigate what impact a change in the distribution of the cylinders has. For this purpose, we consider modules of two adjacent cylinders of different radii $a_{1}$ and $a_{2}$. We choose $a_{1}=0.25,0.5,0.75,1$ and $a_{2}$ accordingly such that the sum of the cross-sectional areas is always equal to $2 \pi$, i.e. $a_{2}=\sqrt{2-a_{1}^{2}}$. Obviously, $a_{1}=1$ implies $a_{2}=1$, i.e. the two cylinders are identical in this case and this is in fact equivalent to a line array of single cylinders with half the spacing, which we consider in the next section.

We choose the line-array spacing $R=6$ and consider the total reflection of a stack of two and eight of such line arrays over a range of incident wavenumbers. The incident angle is $\chi=\pi / 3$ in all cases and the stack spacing in $s_{n}=5$.

It can be seen that the general behaviour of the curves is more or less the same for all cylinder radius distributions, particularly for small wavenumber. This is to be expected since the long waves do not interact as strongly with the objects. It is interesting to note that there is a sharp spike around $k=0.7$ for all cylinder radius distributions except for the case where the cylinders are identical.

7.1.3. Bloch transmission in a fully periodic stack. We want to investigate how well the Bloch transmission approximates the transmission by a finite number of arrays. For this purpose, we consider the setting with eight arrays of identical cylinders from the previous section and compare this to the corresponding Bloch 

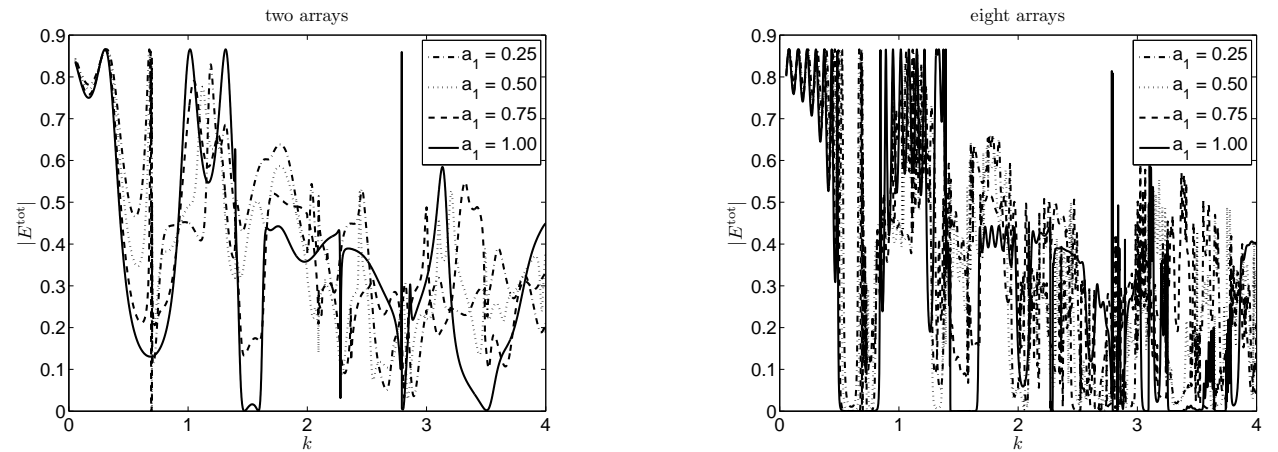

FIG. 7.2. Total transmission (as defined in (5.4)) versus incident wavelength for different cylinder radius distributions for two arrays (left) and eight arrays (right) in the stack.
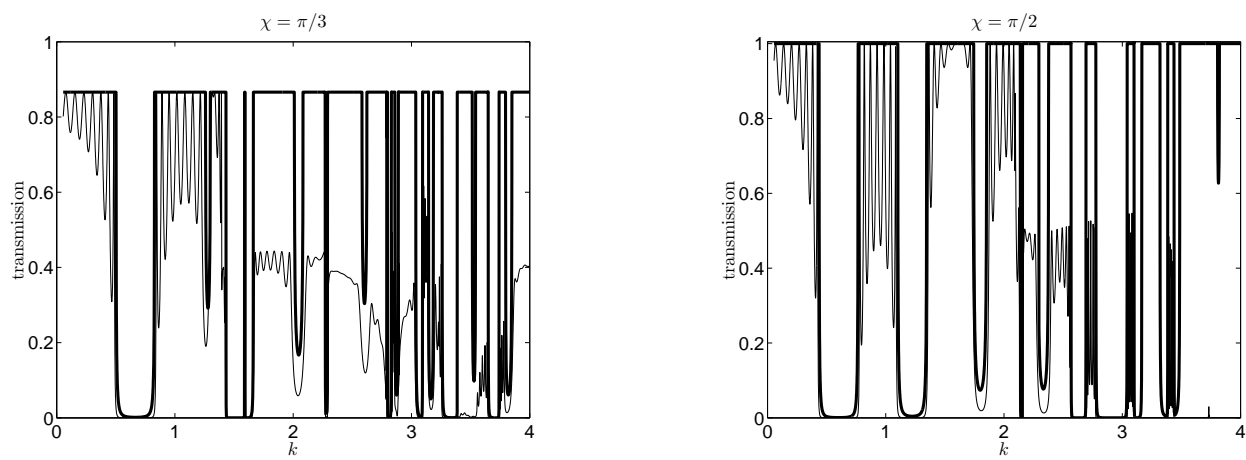

Fig. 7.3. Total transmission as defined in (5.4) for eight arrays in the stack (thin line) and Bloch transmission (thick line) versus incident wavelength for incident angle $\chi=\pi / 3$ (left) and $\chi=\pi / 2$ (right).

transmission. Figure 7.3 (left) shows the total transmission for a stack of eight arrays of cylinders with unit radius compared to the eighth power of the largest eigenvalue of the matrix in (6.6) having absolute value less or equal to one multiplied with $\sin \chi$. The thin curve is obviously identical to the solid curve in figure 7.2 (right). Figure 7.3 (right) shows the analogous results for incident angle $\chi=\pi / 2$.

It can be seen that the Bloch transmission indeed approximates the behaviour of eight arrays quite well. A comparison with a stack of twenty arrays (not shown) gives even better agreement as would be expected.

7.2. Ice floes. The quantity of main interest when considering scattering by ice floes in the MIZ is the total transmitted energy $E^{\text {tot }}$ (cf. (5.4)), and its attenuation. We present some qualitative results for typical parameter sets comparing square and circular ice floes and present a comparison of the attenuation coefficient with experimental data of [32].

The ice floes are modelled as floating elastic plates of shallow draft following [21]. They are determined by the (two-dimensional) region they occupy as well as the 

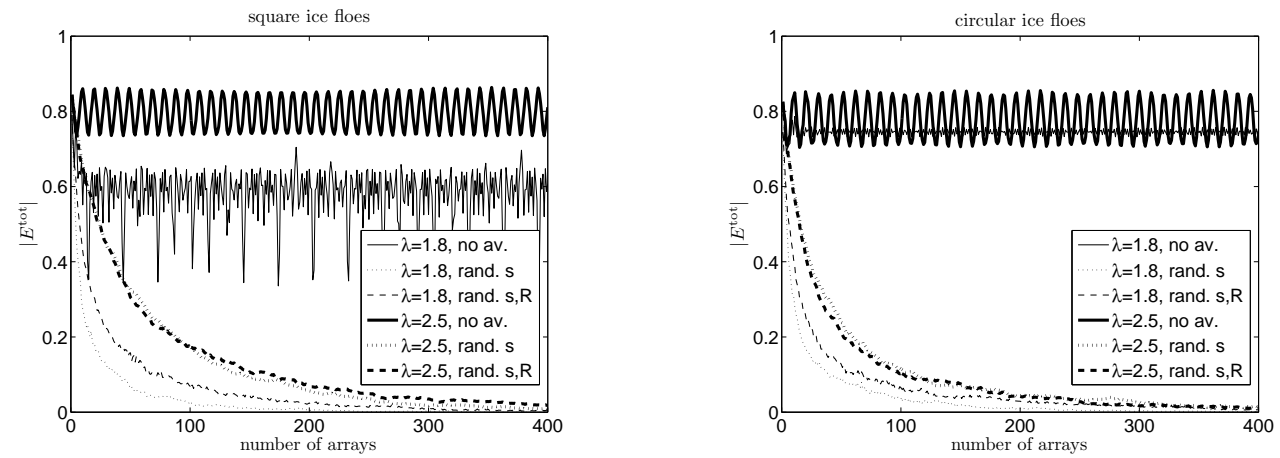

FIG. 7.4. Total transmission versus number of arrays in the stack for incident angle $\chi=\pi / 3$ and two different wavelengths with and without different averaging techniques using square (left) and circular (right) ice floes.

dimensionless stiffness and mass parameters

$$
\beta=\frac{E h^{3}}{12\left(1-\nu^{2}\right)} \frac{1}{g \rho L^{4}} \quad \text { and } \quad \gamma=\frac{\rho_{\mathrm{i}} h}{\rho L},
$$

where $E$ is Young's modulus, $\nu$ is Poisson's ratio, $h$ is the floe thickness, $\rho$ and $\rho_{\mathrm{i}}$ are the density of water and ice, respectively, and $L$ is a characteristic length.

7.2.1. Square and circular ice floes. For incident angle $\chi=\chi / 3$ and two wavelengths $\lambda=1.8,2.5$, we consider scattering by stacks of square and circular ice floes of non-dimensional mass and stiffness 0.02 (in the non-dimensionalisation of [21]). The water depth is taken as 1.5 and basically implies deep water. Figure 7.4 shows the total transmitted energy versus number of arrays in the stack for the periodic arrangement as well as using the different averaging methods described in $\S 6.2$. It can be seen that averaging over random distances between the arrays is necessary in order to obtain an exponential decay of the transmitted energy, which is what is observed in experiments, cf. [32] e.g. Also averaging over the spacing of the arrays only affects the results marginally. It can be noted that solely averaging over array spacings (not shown) does not lead to an exponential decay but to an oscillatory curve similar to that without any averaging. Thus, averaging over distances between arrays is most important, which, serendipitously, is also the least costly.

It can also be observed that the respective curves for square and circular ice floes look more similar to one another for the shorter incident wavelength.

7.2.2. Comparison with experimental data. It is not our purpose here to give an extensive comparison of our model to experimental data. We also note that the experimental data is not extensive, is often missing important information (such as floe size and thickness), lacks consistency (so that experiments performed in similar conditions give quite different results) and are mostly more than thirty years old.

Nevertheless, we compare our simulation results to the experimental data of Squire and Moore [32], which is regarded as one of the best experiments ever performed. Certainly, it is one of the few experiments to have been published in detail, or to have been the subject of an entire paper. Squire and Moore give attenuation coefficients for six dominant wavelengths. The attenuation coefficient is the number $\mathfrak{a}$, for which 


\begin{tabular}{ccccc} 
Wave period $[\mathrm{s}]$ & \multicolumn{5}{c}{ Attenuation $\left[10^{-4} \mathrm{~m}^{-1}\right]$} \\
& Experiment & Sim. 1 & Sim. 2 & Sim. 3 \\
12.2 & $0.272 \pm 0.054$ & 0.0258 & 0.0005 & 0.0011 \\
9.4 & $0.438 \pm 0.036$ & 0.0102 & 0.0032 & 0.0658 \\
7.6 & $0.855 \pm 0.049$ & 0.6310 & 0.2341 & 1.0031 \\
6.4 & $1.087 \pm 0.037$ & 6.2480 & 4.6381 & 11.142 \\
5.5 & $1.214 \pm 0.192$ & 19.392 & 24.153 & 12.825 \\
\multicolumn{5}{c}{ TABLE 7.1}
\end{tabular}

Attenuation coefficient experimentally found by Squire and Moore [32] and simulation results for $0.5 \mathrm{~m}$ thick ice floes of $40 \mathrm{~m}$ side length (simulations 1 and 2) as well as 0.6 thick ice floes of $50 \mathrm{~m}$ side length (simulation 3 ).

the curves in figure 7.4 most closely satisfy

$$
E^{\text {tot }}(M) \approx \mathrm{e}^{-\mathfrak{a} M} \sin \chi .
$$

It turns out that, since $E^{\text {tot }}(M)$ has strong exponential-decay behaviour, a can easily be determined by a least-square fit and the approximation error is very small in all cases considered. We use square ice floes of dimensionless side length 2 for all simulations and we only employ averaging over distances between the arrays in a stack.

We choose the following parameters to account for the experimental conditions: $E=6 \cdot 10^{9} \mathrm{Nm}^{-2}, \nu=1 / 3, \rho=1000 \mathrm{~kg} / \mathrm{m}^{3}$ and $\rho_{\mathrm{i}}=900 \mathrm{~kg} / \mathrm{m}^{3}$. All numerical experiments refer to head-on incidence, i.e. $\chi=\pi / 2$. The body spacing in the line arrays is $R=\sqrt{4 / c}$, where $c$ is the ice concentration given by Squire and Moore as 0.5 . The water depth is two thirds of the wavelength in order to account for deep water.

Table 7.1 shows the (dimensional) attenuation coefficient experimentally found by Squire and Moore together with our simulation results. Simulations 1 and 2 refer to $L=20 \mathrm{~m}$ (i.e. floe side length $40 \mathrm{~m}$ ) and $h=0.5 \mathrm{~m}$, while simulation 3 is for $L=25 \mathrm{~m}$ and $h=0.6 \mathrm{~m}$. For simulation 1, the averaging has been done over a vertical spacing with mean $2 R$ and standard deviation 1.5, while simulations 2 and 3 refer to averaging with mean $R$ and standard deviation $1 / 3$. In all cases, a cut-off was implemented so that ice floes cannot overlap.

The results show that the present method gives attenuation coefficients which are within an order of magnitude. We could fit the data for a given frequency by changing the physical parameters to values which would not be unrealistic. However, the present scattering model shows much greater variation with frequency than was observed in the experiment. This feature was also observed for the simpler twodimensional model [10]. Several reasons for this behaviour are possible and further research is required.

8. Summary and discussion. A very efficient solution method for the waterwave-scattering problem by a large number of bodies has been presented. The method is applicable to arbitrary bodies. Bloch transmission for periodic configurations of bodies as well as averaging techniques for more or less random body arrangements have been discussed. In particular, it has been shown how the method can be used to calculate the attenuation of ocean waves by Marginal Ice Zones.

The presented simulation results for Marginal Ice Zones are rather preliminary but show the potential of the method to build a model Marginal Ice Zone, for which 
simulations are feasible. It is also worth pointing out that the discussed averaging techniques are highly parallelisable although this is probably not necessary. More research needs to be undertaken to find out whether it is sensible to average over other model parameters as well, such as the ice floe geometry, size, material parameters and so on. Moreover, the influence of the particular ice floe model is worth examining. Such investigations will be presented in future publications.

It is noteworthy that, because of the efficiency of the method, it is also easy to incorporate further observed properties of Marginal Ice Zones, such as a changing denseness in the ice cover or a changing thickness of the ice as functions of distance from the ice edge. Moreover, the evolution of the directional spectrum can also be extracted from the simulation results.

Acknowledgements. We would like to thank Luke Bennetts (University of Otago) for the numerous fruitful discussions during this research. This research was supported by Marsden grant UOO308 from the New Zealand government.

\section{REFERENCES}

[1] M. Abramowitz and I.A. Stegun, eds., Handbook of Mathematical Functions, Dover Inc. New York, 1964.

[2] L. G. Bennetts, N. R. T. Biggs, And D. Porter, Wave scattering by an axisymmetric ice floe of varying thickness, IMA J. Appl. Math., (2008). doi:10.1093/imamat/hxn019.

[3] S. Chakrabarti, Hydrodynamic interaction forces on multi-moduled structures, Ocean Engng, 27 (2000), pp. 1037-1063.

[4] R. A. Dalrymple, S. N. Seo, And P. A. Martin, Water wave scattering by rows of circular cylinders, in Proc. Int. 21st Coastal Engng Conf., Malaga, Spain, B. L. Edge, ed., 1988, pp. 2216-2228.

[5] D. V. Evans, The wide-spacing approximation applied to multiple scattering and sloshing problems, J. Fluid Mech., 210 (1990), pp. 647-658.

[6] J.-S. Goo And K. Yoshida, A numerical method for huge semisubmersible responses in waves, SNAME Transactions, 98 (1990), pp. 365-387.

[7] X. Hu and C. T. Chan, Refraction of water waves by periodic cylinder arrays, Phys. Rev. Lett., 95 (2005), p. 154501.

[8] H. Kagemoto And D. K. P. Yue, Interactions among multiple three-dimensional bodies in water waves: an exact algebraic method, J. Fluid Mech., 166 (1986), pp. 189-209.

[9] M. KashiwaGi, Hydrodynamic interactions among a great number of columns supporting a very large flexible structure, J. Fluids Struct., 14 (2000), pp. 1013-1034.

[10] A. Kohout and M. H. Meylan, An elastic plate model for wave attenuation and ice floe breaking in the marginal ice zone, J. Geophys. Res., 113 (2008).

[11] C. M. Linton, The Green's function for the two-dimensional Helmholtz equation in periodic domains, J. Engng. Math., 33 (1998), pp. 377-402.

[12] C. M. Linton And D. V. Evans, The interaction of waves with a row of circular cylinders, J. Fluid Mech., 251 (1993), pp. 687-708.

[13] C. M. Linton and P. McIver, Handbook of Mathematical Techniques for Wave / Structure Interactions, Chapman \& Hall /CRC, 2001.

[14] C. M. Linton and I. Thompson, Resonant effects in scattering by periodic arrays, Wave Motion, 44 (2008), pp. 165-175.

[15] P. A. Martin, Multiple scattering. Interaction of time-harmonic waves with $N$ obstacles, Cambridge University Press, 2006.

[16] D. Masson And P. H. LeBlond, Spectral evolution of wind-generated surface gravity waves in a dispersed ice field, J. Fluid Mech., 202 (1989), pp. 111-136.

[17] P. MCIver, Water-wave propagation through an infinite array of cylindrical structures, J. Fluid Mech., 424 (2000), pp. 101-125.

[18] - Wave interaction with arrays of structures, Appl. Ocean Res., 24 (2002), pp. 121-126.

[19] R. C. McPhedran, L. C. Botten, A. A. Asatryan, N. A. Nicorovici, C. M. de Sterke, And P. A. Robinson, Ordered and disordered photonic band gap materials, Aust. J. Phys., 52 (1999), pp. 791-809.

[20] R. C. McPhedran, L. C. Botten, A. A. Asatryan, N. A. Nicorovici, P. A. Robinson, 
AND C. M. DE SteRKe, Calculation of electromagnetic properties of regular and random arrays of metallic and dielectric cylinders, Phys. Rev. E, 60 (1999), pp. 7614-7617.

[21] M. H. Meylan, Wave response of an ice floe of arbitrary geometry, J. Geophys. Res. - Oceans, 107 (2002), p. doi: 10.1029/2000JC000713.

[22] M. H. Meylan And D. Masson, A linear Boltzmann equation to model wave scattering in the marginal ice zone, Ocean Modelling, 11 (2006), pp. 417-427.

[23] M. H. Meylan, V. A. Squire, and C. Fox, Towards realism in modeling ocean wave behavior in marginal ice zones, J. of Geophysical Research, 102 (1997), pp. 22981-22991.

[24] L. S. Mulholland and M. A. Heckl, Multi-directional sound wave propagation through a tube bundle, J. Sound Vibration, 176 (1994), pp. 377-398.

[25] W. Perrie and Y. Hu, Air-ice-ocean momentum exchange. part 1:energy transfer between waves and ice floes, J. of Phys. Ocean., 26 (1996), pp. 1705-1720.

[26] M. A. Peter and M. H. Meylan, Infinite-depth interaction theory for arbitrary floating bodies applied to wave forcing of ice floes, J. Fluid Mech., 500 (2004), pp. 145-167.

[27] — Water-wave scattering by a semi-infinite periodic array of arbitrary bodies, J. Fluid Mech., 575 (2007), pp. 473-494.

[28] — Water-wave scattering of vast fields of bodies such as ice floes in the Marginal Ice Zone, in Proc. 24th Int. Workshop on Water Waves and Floating Bodies, Zelenogorsk, Russia, A. Korobkin and P. Plotnikov, eds., 2009. in press.

[29] M. A. Peter, M. H. Meylan, and H. Chung, Wave scattering by a circular elastic plate in water of finite depth: a closed form solution, IJOPE, 14 (2004), pp. 81-85.

[30] M. A. Peter, M. H. Meylan, and C. M. Linton, Water-wave scattering by a periodic array of arbitrary bodies, J. Fluid Mech., 548 (2006), pp. 237-256.

[31] R. Porter and D. V. Evans, Rayleigh-Bloch surface waves along periodic gratings and their connection with trapped modes in waveguides, J. Fluid Mech., 386 (1999), pp. 233-258.

[32] V.A. Squire and S. C. Moore, Direct measurement of the attentuation of ocean waves by pack ice, Nature, 283 (1980), pp. $365-368$.

[33] V. A. Squire, Of ocean waves and sea-ice revisited, Cold Regions Science and Technology, 49 (2007), pp. 110-133.

[34] V. A. Squire, J. P. Dugan, P. Wadhams, P. J. Rottier, and A. J. Liu, Of ocean waves and sea ice, Annu. Rev. Fluid Mech., 27 (1995), pp. 115-168.

[35] V. TwERsKy, On scattering of waves by the infinite grating of circular cylinders, IRE Trans. on Antennas and Propagation, 10 (1962), pp. 737-765.

[36] P. Wadhams, V. A. Squire, D. J Goodman, A. M. Cowan, and S. C. Moore, The attenuation rates of ocean waves in the marginal ice zone, J. Geophys. Res., 93 (1988), pp. $6799-6818$.

[37] C. D. WAng, M. H. Meylan, And R. Porter, The linear wave response of a periodic array of floating elastic plates, J. Engng Math., 57 (2007), pp. 23-40. 